\title{
Enunciación
}

\section{Concepciones docentes: reflexiones sobre las prácticas en la enseñanza de la escritura}

\author{
Teaching conceptions: Reflections about practices in the teaching of writing
}

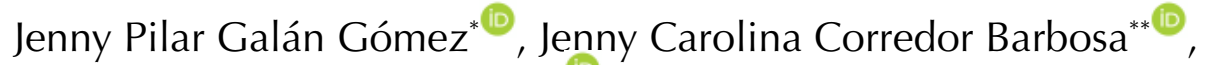 \\ Viviana Marcela López Contreras ${ }^{* * * \mathbb{C}}$, Yolanda Milena Soto Valderrama*
}

\begin{abstract}
Resumen
Este artículo presenta los resultados de la aplicación de una serie de talleres realizados en dos instituciones educativas distritales, que tuvieron como propósito analizar las concepciones que tienen algunos docentes de básica y media, frente a los procesos de enseñanza y aprendizaje de la escritura. Así mismo, promover una reflexión en torno a sus prácticas pedagógicas en relación con la modalidad escrita de la lengua. La metodología empleada se orientó desde el enfoque cualitativo y el diseño que sirvió de base en la aplicación de los talleres fue la investigación-acción. Por su parte, los talleres se caracterizaron por la posibilidad de diálogo, en la que los docentes comparten sus experiencias y opiniones, negocian frente a las propuestas y participan en la evaluación de este. Los hallazgos evidencian que, a pesar del conocimiento de los docentes sobre diferentes estrategias para la enseñanza y aprendizaje de la escritura, en sus prácticas predominan metodologías tradicionales desde un enfoque prescriptivo, que centra su atención en un solo aspecto de la enseñanza de la lengua, opuesto a lo que se propone desde los Lineamientos Curriculares de Lengua Castellana, donde se concibe la escritura como un proceso dinámico y significativo que cumple una función social. En conclusión, se podría afirmar que es necesario propiciar espacios para la reflexión sobre la enseñanza y aprendizaje de la escritura a partir del análisis del quehacer docente en el aula.
\end{abstract}

Palabras clave: enseñanza de la escritura, aprendizaje, prácticas en enseñanza.

\begin{abstract}
This article presents the results of the application of several workshops carried out in two public education institutions, with the aim of analyzing the conceptions that elementary and basic education teachers have regarding teaching and learning processes of writing. The purpose was also to promote a reflection on the pedagogical practice in relation to the written modality of the language. The methodology used was oriented from the qualitative approach and the design of the workshops was characterized by the possibility of establishing a spontaneous dialogue with teachers, sharing experiences and opinions, negotiating and evaluating the proposals. The findings show that, despite the knowledge of teachers about different strategies to teaching and learning of writing, their practices focus on traditional methodologies from a prescriptive approach and only pay attention to one aspect of language
\end{abstract}

* Docente de Lengua Castellana, SED, Colegio Alexander Fleming. Licenciada en Educación Básica con énfasis en Español e Inglés de la Universidad Pedagógica Nacional. Magíster en Pedagogía de la Lengua Materna de la Universidad Distrital Francisco José de Caldas. Correo electrónico: ypgalang@correo.udistrital.edu.co

** Licenciada en Educación Básica con énfasis en Humanidades y Lengua Castellana de la Universidad Distrital Francisco José de Caldas. Especialista en Didácticas para Lectura y Escritura con énfasis en Literatura de la Universidad de San Buenaventura. Buenaventura. Docente SED, Colegio Tibabuyes Universal. Magíster en Pedagogía de la Lengua Materna de la Universidad Distrital Francisco José de Caldas. Correo electrónico: jccorredorb@correo.udistrital.edu.co

*** Licenciada en Pedagogía Infantil de la Universidad Distrital Francisco José de Caldas. Especialista en Procesos Lectoescriturales de la Corporación Educativa Minuto de Dios. Magíster en Pedagogía de la Lengua Materna de la Universidad Distrital Francisco José de Caldas. Correo electrónico: vmlopezc@correo.udistrital. edu.co

**** Docente de básica primaria, SED, Colegio Provincia de Quebec. Licenciada en Educación con énfasis en Educación Especial de la Universidad Pedagógica Nacional. Especialista en Gerencia de Proyectos Educativos Institucionales y maestrante en Pedagogía de la Lengua Materna de la Universidad Distrital Francisco José de Caldas. Correo electrónico: ymsotov@correo.udistrital.edu.co

Cómo citar: Galán Gómez, J. P., Corredor Barbosa, J. C., López Contreras, V. M. y Soto Valderrama, Y. M. (2020). Concepciones docentes: reflexiones sobre las prácticas en la enseñanza de la escritura. Enunciación, 25(2), 232-246. https://doi.org/10.14483/22486798.15422

Artículo recibido: 18 de octubre de 2019; aprobado: 1 de julio de 2020 
teaching, opposed to what is proposed in the political documents where writing is conceived as a dynamic and significant process that has a social function. In conclusion, it could be affirmed that it is necessary to promote reflection about teaching and learning of writing from the analysis of the teacher's task in the classroom.

Keywords: writing teaching, learning, teaching practice.

\section{Introducción}

La escritura como actividad discursiva es una habilidad compleja que involucra diferentes procesos para producir un texto; es decir, un escritor realiza una serie de operaciones cognitivas, lingüísticas y socioculturales que le permiten la construcción de un escrito que responda a una necesidad comunicativa real. En concordancia con esta idea en los Lineamientos Curriculares de Lengua Castellana (MEN, 1998):

[...] la escritura se define como un proceso social e individual en el que se configura un mundo y se ponen en juego saberes, competencias, intereses, y que a la vez está determinado por un contexto sociocultural y pragmático que determina el acto de escribir: escribir es producir el mundo. (p. 49)

En este mismo sentido, y de acuerdo con Cassany (1999), la escritura es tanto un proceso como una habilidad que exige una demanda de operaciones cognitivas y discursivas por parte del escritor, quien debe generar ideas, organizarlas, identificar la audiencia, revisar el texto y evaluarlo.

Sin embargo, hoy es común encontrar en la escuela y en las prácticas de aula que la escritura se concibe como la mera habilidad de codificación, en la cual prima el manejo del código alfabético, es decir, tiene un uso prescriptivo que carece de sentido. Por esta razón su enseñanza sigue siendo tradicional, pues se reduce al conocimiento de fonemas y grafemas sin una relación de significación que permita reconocer su función social. De esta manera se considera necesario y pertinente reflexionar sobre el quehacer docente y las concepciones que subyacen en los procesos de enseñanza y aprendizaje de la escritura, con el propósito de determinar desde qué perspectivas teóricas se orienta dicho proceso y cuál es la incidencia que tiene en la formación de los estudiantes.

Generar un espacio de diálogo con docentes de diferentes instituciones posibilita la identificación de las problemáticas relacionadas con la enseñanza de la lengua escrita en los primeros años de escolaridad, a partir de la reflexión y la autoevaluación se pueden generar soluciones o alternativas que contribuyan a fortalecer este proceso de una manera significativa, reconociendo además la importancia del rol docente en esta etapa de formación.

Así, surge el interés del grupo de investigación Oralitura, adscrito a la Maestría en Pedagogía de la Lengua Materna de la Universidad Distrital Francisco José de Caldas, de realizar el diseño, aplicación y evaluación de talleres dirigidos a docentes de básica primaria, secundaria y media de dos instituciones públicas de Bogotá. El propósito estuvo orientado a analizar las concepciones que tienen frente a los procesos de enseñanza y aprendizaje de la escritura, y con ello, promover una reflexión en torno a sus prácticas pedagógicas para reconocer tanto las fortalezas como los aspectos por mejorar, y así, de acuerdo con los contextos particulares, establecer estrategias que contribuyan al enriquecimiento de dichas prácticas a partir del intercambio de experiencias. Para la aplicación de estos talleres se contó con la participación de nueve docentes del Colegio Provincia de Quebec, ubicado en la localidad 5 Usme y nueve docentes del Colegio Alexander Fleming, de la localidad 18 Rafael Uribe Uribe.

\section{Marco teórico}

Este apartado lo constituye el abordaje y análisis teórico de diversas categorías que emergen de la 
relación entre la enseñanza y el aprendizaje de la escritura, con las concepciones y prácticas docentes en el aula. Las categorías que aquí se desarrollan surgen de las necesidades encontradas en los contextos educativos, en los que se desenvuelven las integrantes del grupo de investigación, de la revisión de los documentos políticos y la preocupación sobre cómo aprenden a escribir los estudiantes. De igual manera, se tiene en cuenta la fundamentación teórica y los estudios etnográficos que evidencian una multiplicidad de limitaciones en la enseñanza y aprendizaje de la escritura.

\section{Concepciones y prácticas docentes para la enseñanza de la escritura}

En primera medida, es necesario precisar qué entiende el grupo de investigación Oralitura por concepción. Para ello, se toma la definición dada por las docentes investigadoras de la Universidad Distrital Francisco José de Caldas, en su artículo "Relación entre concepciones sobre el lenguaje y la lengua escrita":

Las concepciones son una red semántica de conocimientos construida por el individuo, la cual está edificada sobre la práctica social y cultural que genera la sociedad. No son simplemente una producción mental sino que más bien corresponden a un proceso que resulta de la elaboración de sentido que se construye en las interacciones lingüísticas. (Morales y Bojacá, 2000, p. 109)

Si se parte de esta definición, entonces las concepciones docentes sobre la enseñanza y aprendizaje de la escritura están basadas en una multiplicidad de redes; son producto tanto de las experiencias personales como de los procesos individuales de adquisición del código, y están influenciadas por la formación como profesionales del lenguaje. Ahora bien, la escritura en cuanto proceso, que implica la materialización de un constructo social proveniente de la lengua oral, requiere además que su enseñanza esté orientada desde planteamientos teóricos y metodológicos sólidos para su correcto desarrollo. En este sentido, cabe señalar que aquellas prácticas docentes desprovistas de alguna reflexión solamente perpetúan una concepción docente de la enseñanza de la escritura carente de sentido y transformación en la escuela.

Pese a que los docentes reconocen la importancia de la escritura en el desarrollo social del individuo, su enseñanza dista de las múltiples teorías, producto de las diversas reflexiones y análisis de los últimos años. Al respecto, la licenciada e investigadora Delia Lerner, en su libro Leer y escribir en la escuela: lo real, lo posible y lo necesario (2001), afirma que "enseñar a leer y escribir es un desafío que trasciende ampliamente la alfabetización en sentido estricto. El desafío que hoy enfrenta la escuela es el de incorporar a todos los alumnos a la cultura de lo escrito" (p. 25). Por tanto, no puede quedarse solamente en el aprendizaje del código escrito, sino que debe comprenderse como un proceso que se desarrolla en interacción con otros y con una intención particular.

La autora hace referencia a que en la realidad se presentan distintas dificultades en la enseñanza de la escritura, relacionadas con las concepciones del docente frente a la didáctica de la lengua escrita. Una de estas dificultades corresponde a los objetivos planteados para escribir en la escuela, porque generalmente no responden a propósitos comunicativos reales. Dicho de otra manera, el docente considera poco relevante el contexto en este proceso e implementa una serie de actividades generalmente desligadas de los intereses y necesidades de los estudiantes.

Por otro lado, se encuentra que la enseñanza de la escritura se aborda de manera fragmentada; es decir, se enseña la lengua escrita a partir de las partes que la componen. En consecuencia, se lleva al estudiante a especializarse en las unidades mínimas de la lengua como la escritura de palabras y frases carentes de significación y relevancia. Esto ocurre porque el docente supone, como lo afirma Kenneth Goodman (1990), que: 
Al dividir el lenguaje natural en fragmentos de tamaño de bocadillo, pequeños pero abstractos. Como parecía lógico pensar que los niños aprendían mejor cositas sencillas, desarmamos el lenguaje y lo convertimos en palabras, sílabas y sonidos aislados. Desafortunadamente, también desechamos su propósito natural -la comunicación del significado- y convertimos el lenguaje en una serie de abstracciones, sin relación con las necesidades y experiencias de los niños a quienes pretendíamos ayudar. (p. 78)

Es decir, considerar la enseñanza de la escritura como desarrollo de la habilidad de codificación limita la posibilidad de los aprendices de reconocer en ella el valor social que cumple. La concepción del docente influye en la adquisición y desarrollo de la escritura tanto en edades tempranas como en los últimos grados de formación en la escuela.

Otra dificultad que se reconoce en las prácticas educativas es la poca importancia que se le da al proceso de composición o elaboración como al propósito comunicativo de la escritura; en cambio, se le otorga trascendencia a la evaluación a través de la calificación de un producto, en el cual se tienen en cuenta aspectos prescriptivos: "la ortografía de las palabras ocupa en la enseñanza un lugar más importante que otros problemas más complejos involucrados en el proceso de escritura" (Lerner, 2001, p. 31). Así, el estudiante descuida en algunos casos la totalidad del contenido mismo para preocuparse por la forma desprovista de todo sentido y relevancia.

En este caso, el docente presume que debe enseñar la escritura de la misma manera en la que aprendió, de allí que Goodman (1990) asevere que el propósito de la enseñanza es "enseñar el lenguaje por sí mismo y se obliga a los niños a analizarlo como lo haría un lingüista, el alumno deja de prestar atención a lo que intenta decir o entender a través del lenguaje" (p. 80). En consecuencia, el docente termina cumpliendo el papel más abarcador en el aula, se concibe como el único ser poseedor de conocimiento y se relega al estudiante, quien pasa a ser un agente pasivo de su proceso de aprendizaje. Esto se relaciona con la última dificultad, referida a la evaluación, donde tanto el rol del docente como del estudiante se mantiene desde una mirada tradicional, pues su objetivo es la verificación del aprendizaje. De acuerdo con lo anterior, Lerner (2001) parte del ejemplo del derecho a evaluar:

[...] dado que este derecho es en general privativo del docente, los alumnos tienen muy pocas oportunidades de autocontrolar lo que comprende al leer y de autocorregir sus escritos. Aprender a hacerlo y conquistar autonomía como lectores y escritores resulta entonces muy difícil. (p. 31)

Por consiguiente, se evidencia que la concepción del docente frente a la enseñanza de la escritura influye en su accionar en el aula. En consecuencia, es importante analizar dichas concepciones con el fin de caracterizarlas, generar reflexiones y así propiciar el mejoramiento y la transformación de las prácticas pedagógicas.

\section{La enseñanza de la escritura desde una perspectiva sociocultural}

En el presente artículo se asume la escritura desde la perspectiva sociocultural, por tanto, se define como un proceso que se da a partir de las interacciones del individuo con su medio y en los intercambios que tiene desde su nacimiento, lo cual le permite relacionarse con otros, es decir, requiere de un aprendizaje contextualizado. En este mismo sentido, se entiende como una función psicológica superior avanzada que responde a una necesidad de comunicación con una intención particular que implica reconocer al interlocutor. "Los procesos psicológicos superiores surgen históricamente en las relaciones entre seres humanos. El niño adquiere las formas culturales ya elaboradas en sus relaciones con el adulto y después, también en la relación con sus pares" (Braslavsky, 1997, p. 22).

Desde esta perspectiva, la escritura es aprendida en constante relación con otros, por esta 
razón el papel del docente es fundamental, pues es quien debe fomentar interacciones entre los estudiantes: "El hecho de que las situaciones de colaboración favorezcan el aprendizaje lectoescritor ha de propiciar, por parte del maestro, la propuesta de actividades auténticamente colaborativas, cuyo resultado no sea la suma de actuaciones conjuntas independientes" (Díez, 2004, p. 34), por lo que resulta indispensable que su enseñanza se presente de una manera contextualizada y que responda a una necesidad real de comunicación que evidencie su función en la sociedad.

En el proceso de enseñanza/aprendizaje de la lengua escrita se concibe al niño como el actor principal, sus intereses y motivaciones le permiten seguir descubriendo y buscando respuestas mientras aprende. En este caso a comprender la lengua escrita, al escribir lo hacemos en comunidad, pues el escribir refleja un diálogo que ha surgido de un contexto determinado (Fons, 2012, s.p.); es decir, es él quien descubre y aprende lo que requiere para hacer las actividades; es importante mencionar que el docente, más allá de resolver dudas, se encarga de incentivarlo a resolverlas por sí mismo. Allí aparece la idea de docente/mediador, entendida como el encargado de establecer relaciones entre los estudiantes y el conocimiento, así se permite que ellos aprendan a partir de la interacción que tienen con el saber y con sus pares. De acuerdo con esto, Braslavsky (2003) señala que "se debe enseñar de un modo en que la escritura sea necesaria para el niño, es decir, sentida por él como una tarea virtual imprescindible que debe ser provocada como una necesidad natural" (p. 56). Por tanto, enseñar de forma contextualizada es imprescindible, debido a que se facilita la comprensión de la función de la lengua como actividad discursiva y no solo como un sistema de representación.

Asimismo, no debe desligarse la importancia del aprendizaje de la escritura y su papel en la consolidación como sociedad. Por consiguiente, el docente además de su compromiso pedagógico y disciplinar debe reconocer la trascendencia de la enseñanza de la escritura desde su función social. Frente a la evaluación de la escritura, es formativa porque entre las características están el intercambio de información, la participación de los estudiantes, la negociación y cogestión, las relaciones de comunicación, el diálogo y respeto (Pérez et al., 2009). Al ser concebida la enseñanza situada, la evaluación de igual forma debe responder a una situación real, por lo que se habla de la evaluación auténtica, definida como aquella que se da en un contexto real: "[...] va un paso más allá en el sentido de que destaca la importancia de la aplicación, de la habilidad en el contexto de una situación de la vida real" (Díaz, 2005, s.p.). Con ella se busca la aplicación de las habilidades en la resolución de situaciones reales que le permitan al estudiante reconocer tanto sus fortalezas como los aspectos por mejorar.

\section{Metodología}

\section{Enfoque cualitativo}

Para este trabajo se contó con la participación de 18 docentes de dos colegios distritales de Bogotá, con los cuales se implementaron dos talleres titulados "Taller de concepciones docentes sobre la enseñanza de la lengua materna". A partir de ello, se recogió la información que constituyó un corpus que fue analizado a través de la sistematización, y que está basado en la investigación cualitativa; esta "se orienta a analizar casos concretos en su participación temporal y local, y a partir de las expresiones y actividades de las personas en sus contextos locales" (Flick, 1998, p. 27). De esta manera, no se trata de generalizar las concepciones de los docentes frente a la enseñanza y aprendizaje de la escritura, sino, por el contrario, de analizar desde un contexto particular dichas concepciones para generar un espacio de reflexión que conduzca paulatinamente a una transformación sobre el quehacer docente. 


\section{Diseño: investigación-acción}

El diseño seleccionado fue la investigación-acción, ya que no solo se pretende comprender la realidad estudiada, sino también intervenir en ella para lograr transformaciones. En este caso, con la aplicación del primer taller se buscó la exploración, el reconocimiento y el análisis de las concepciones docentes, con el fin de identificar su incidencia en el proceso de enseñanza de la escritura.

Luego de este primer acercamiento, se implementó el segundo taller, en el cual los docentes participantes conceptualizaron y caracterizaron las diversas teorías de enseñanza y aprendizaje de la lengua. Lo anterior permitió abrir un espacio para la reflexión, cuyo propósito fue establecer una relación/comparación de estas teorías de enseñanza con sus propias prácticas. Por consiguiente, en el segundo y último taller, las investigadoras implementaron una clase/modelo diseñada desde los postulados del enfoque sociocultural, mediante el cual se pretendió evidenciar las ventajas de este enfoque en la enseñanza de la escritura, con el fin de que los docentes participantes analizaran y deconstruyeran sus propias prácticas.

De esta manera, a partir de lo señalado por Elliot (2000), la investigación-acción en la escuela se caracteriza entre otros aspectos, por analizar las acciones humanas y las situaciones sociales experimentadas por los profesores, que pueden ser inaceptables o problemáticas, susceptibles de cambio o que requieren una respuesta práctica. El propósito de este diseño de investigación es profundizar la comprensión del docente sobre el problema planteado, lo que permite formular acciones adecuadas que faciliten llegar a la solución de este. En el mismo sentido, para Contreras (1994), "la investigación-acción es una forma de entender el oficio docente que integra la reflexión y el trabajo intelectual en el análisis de las experiencias que se realizan, como un elemento esencial de lo que constituye la propia actividad educativa" (p. 12).

De acuerdo con esto, se consideró pertinente asumir la investigación-acción como enfoque metodológico para el diseño e implementación de los talleres. Cabe recordar que el ciclo de la investigación-acción, modelo propuesto por Kemmis (1989), se centra en cuatro fases cíclicas: planificación de la acción, acción, observación de la acción y reflexión. Así, el objetivo central de esta investigación consistió en el diseño e implementación de tres talleres, lo cual permitió analizar mediante la observación las concepciones docentes que subyacen a los procesos de enseñanza y aprendizaje de la lengua escrita, propiciando un espacio de reflexión sobre el quehacer docente a través del intercambio de experiencias y su análisis, lo que de manera implícita conlleva a repensar y transformar las prácticas educativas.

A continuación, se presenta una descripción detallada del desarrollo de cada una de las fases del proceso de investigación-acción llevado a cabo por el grupo de investigación Oralitura.

\section{Fase de planificación de la acción}

En un principio surge el interés de las investigadoras por promover la reflexión entre los docentes frente a sus prácticas en el aula con relación a los procesos de enseñanza y aprendizaje de la lengua materna, específicamente, la enseñanza de la escritura. Así, el grupo de investigación Oralitura plantea el desarrollo del taller como propuesta didáctica que permite el intercambio de experiencias entre docentes y, a su vez, la indagación de nuevos modelos pedagógicos que enriquezcan sus prácticas pedagógicas. En palabras de Rodríguez (2012), el taller propicia

[...] el diálogo entre los participantes, exposición libre de los puntos de vista para la negociación de las acciones a seguir, la definición de los propósitos comunes, las funciones de los miembros, las metas por alcanzar y los medios requeridos para lograrlo. (p. 16)

De esta manera se hace énfasis en la importancia de la interacción y el diálogo constantes entre el docente y los demás participantes. Por ello fue 
necesario abordar el taller desde la dimensión lingüístico-discursiva, que parte del desarrollo de la oralidad. Tal proceso se basa en la producción de enunciados que se enfoquen en argumentar, dar soluciones a los distintos problemas y defender sus puntos de vista, como también en la construcción colectiva de saberes que favorezcan el aprendizaje de todos los participantes. También es importante que se generen diálogos que les permitan trabajar en equipo.

La permanente comunicación que se establece entre el docente y los participantes es una gran oportunidad de aprendizaje, pues permite el intercambio de saberes y la construcción colectiva de conocimiento. Teniendo en cuenta la premisa de que el ser humano es un ser social y construye conocimiento a partir del constante diálogo con el otro, el taller coincide con los postulados de Vygotsky sobre el aprendizaje colaborativo:

En el enfoque neo-vigotskiano del aprendizaje colaborativo, el valor de la experiencia sociocomunicativa no radica solo en el acceso a una pluralidad de perspectivas, sino en los beneficios que implica la coordinación social en sí misma: el andamiaje y la ayuda mutua, la estimulación recíproca, la ampliación del campo de acción o de representación, la complementación de roles y el control intersujetos de los aportes y de la actividad. (Roselli, 2016, p. 227)

El docente cumple un rol activo en el desarrollo del taller, es quien guía y orienta cada una de las actividades a través de las preguntas y el diálogo constante con los asistentes. Por su parte, los asistentes participan y de ellos mismos surgen los insumos para el desarrollo y el cumplimiento del propósito del taller. Desde la perspectiva sociocultural, se sustenta el desarrollo de los talleres a partir de la interacción y de la participación de los aprendices, que en este caso son los docentes. En este sentido, se define los objetivos general y específicos de la investigación, los cuales se enmarcan en la fase de acción, observación de la acción y reflexión.

\section{Fase de acción y observación de la acción}

Talleres de concepciones docentes sobre la enseñanza de la lengua materna

Cada taller se desarrolló en tres grandes momentos, dispuestos en forma de espiral a saber: sensibilización, fundamentación y socialización-evaluación. Con el fin de que tanto los docentes participantes como quienes los aplicaron no perdieran de vista los objetivos que se trazaron en un principio. Los talleres contaron con la autoevaluación, en la que se logró el reconocimiento de las habilidades adquiridas a través de múltiples reflexiones que permitieron la construcción y/o replanteamiento de algunas concepciones.

\section{Taller 1}

Los objetivos de este taller se enfocaron en identificar y describir las prácticas pedagógicas de los docentes. A partir de ello, se dio paso a un breve recorrido histórico sobre las teorías que se han desarrollado en relación con la enseñanza y aprendizaje de la escritura. A continuación se presenta una breve descripción de cada uno de los momentos.

El primer momento se denominó fase de sensibilización. En esta se buscó generar motivación e interés por parte de los docentes. Con ello se logró activar sus conocimientos previos, las experiencias personales y profesionales frente a la enseñanza y aprendizaje de la escritura. Cabe mencionar que la información que proporcionaron los profesores en esta parte se tomó como insumo para establecer relaciones con las actividades posteriores.

Este momento en el primer taller se denominó "Explorando nuestros conocimientos previos". Su intención fue llevar a los docentes a evocar y compartir un recuerdo sobre cómo aprendieron ellos a escribir. Cada docente relató, desde su experiencia personal, cómo le fue enseñada la escritura en su etapa preescolar y, con ello, los aspectos que los marcaron significativamente. Luego se indagó para saber si estas formas de enseñanza aún continuaban siendo vigentes en las prácticas docentes actuales. Además se les preguntó qué incidencia 
tenían estas experiencias en el proceso de formación de los estudiantes. Luego, se cerró con una reflexión en torno a las ventajas y desventajas de dichas estrategias.

En el segundo momento, "Compartiendo experiencias", a cada docente se le entregó una hoja que contenía un registro de clase de una sesión de lengua castellana con estudiantes del grado séptimo de una institución educativa. Se les indicó a los docentes que además de leer y analizar dicho registro, lo relacionaran en una tabla que les permitiera identificar cuál había sido el rol del docente, el de los estudiantes y el objeto de enseñanza. Por último, los participantes socializaron las impresiones que les dejó este registro, con respecto a las prácticas docentes y su incidencia en el aprendizaje de la escritura por parte de los estudiantes. Esta actividad se implementó con el fin de identificar cuáles eran las concepciones de los docentes frente a los diversos roles que cumplen los maestros y los estudiantes en aula y sus apreciaciones sobre el objeto de enseñanza.

El segundo momento se constituyó en un tránsito entre las concepciones del docente y los conceptos basados en diversas teorías, la fundamentación. Se inició con una caracterización de las teorías de enseñanza y aprendizaje de la lengua escrita y los modelos pedagógicos relacionados con estas. En el tablero se realizó un cuadro comparativo en el que se ubicó el nombre de las teorías, las características sobresalientes de cada modelo pedagógico, en las que se destacaron los roles tanto del docente como del estudiante en el proceso de enseñanza y aprendizaje. Para la elaboración de este cuadro se contó con la participación de los docentes quienes, desde su saber, aportan a la construcción de conocimiento. Durante este momento las investigadoras hicieron las aclaraciones y precisiones pertinentes sustentadas en la caracterización de los modelos epistemológicos, pedagógicos y lingüísticos en la enseñanza de la lengua materna, que realiza Jaimes (2008) en su libro Aprender en el aula de preescolar. Lo anterior permitió sentar las bases sobre las teorías y concepciones de enseñanza y aprendizaje de la lengua que fundamentan las prácticas pedagógicas del lenguaje.

Para el momento de cierre, socialización y evaluación, se les pidió a los docentes que, teniendo en cuenta cada una de las actividades realizadas en los tres momentos del taller y principalmente de acuerdo con los conocimientos construidos en la fundamentación, respondan y socialicen sus respuestas frente a las siguientes preguntas: ¿Qué es la escritura?, ¿para qué se enseña a escribir?, y ¿cómo se debería enseñar a escribir en el aula? Este momento tuvo como propósito identificar, por un lado, cuáles eran las dificultades que se presentaron durante el desarrollo del taller, así como conocer las dudas y las preguntas frente a los conceptos trabajados en relación con la enseñanza de la escritura, y por otro lado, reconocer cuáles son los aprendizajes que les dejó el taller.

\section{Taller 2}

$\mathrm{Al}$ igual que en el taller 1, el primer momento lleva a los docentes a recordar una práctica pedagógica propia, considerada exitosa. Aquí, se realizó la socialización de una experiencia significativa de enseñanza de la lengua escrita por parte de los docentes. Se les pidió que tuvieran en cuenta para su descripción de esta clase, el objetivo que se buscaba, la población a la cual estuvo dirigida y por qué se consideró exitosa dicha práctica.

Esta fase se denominó "Compartiendo experiencias", y buscó fomentar la reflexión de los docentes frente a sus prácticas pedagógicas en la enseñanza de la escritura. En un intercambio de experiencias, cada uno describió una clase y explicó por qué consideraba que había sido exitosa, se presentó en un cuadro el rol del docente, el rol del estudiante y el objeto de enseñanza de cada uno. Al hacer la socialización con los demás docentes se lograron concretar cuáles habían sido las fortalezas y debilidades de cada práctica, de manera que esta pudiera ser enriquecida.

"La práctica hace al maestro" fue el siguiente momento del taller 2. En este se implementó una 
clase modelo con los docentes desde la perspectiva sociocultural, con el fin de que ellos reconocieran los aportes del modelo interaccionista en la enseñanza y aprendizaje de la escritura. Para dar inicio a esta clase, se les pidió a los docentes que respondieran qué conocimientos tenían sobre el texto instructivo, asimismo todo aquello que quisieran aprender sobre este tipo de texto. Luego, se compartió con los docentes, por un lado, el objetivo que se pretendía alcanzar con la implementación de esta clase y, por otro lado, su propósito interno.

De esta manera, para el desarrollo de la clase se propuso elaborar una figura hecha en origami mediante la identificación de la función comunicativa del texto instructivo. Primero, se les pidió a los docentes que se organizaran por grupos y escogieran una figura de origami que conocieran previamente, con el fin de escribir un texto instructivo sobre el proceso que se debe seguir para su elaboración. Posteriormente, los textos construidos por cada grupo de docentes fueron intercambiados entre sí, para que, mediante la lectura de estas instrucciones, ellos elaboraran la figura siguiendo los pasos propuestos. Por último, se realizó una socialización en la cual se dio a conocer de forma dialogada cómo se sintieron durante el desarrollo actividad, si lograron cumplir con la elaboración de la figura y cuáles fueron las dificultades presentadas con el texto de sus compañeros y la funcionalidad de este.

En el cuarto momento, "Socializar y reflexionar", se buscó reconocer y analizar cuáles fueron las dificultades frente a la elaboración del texto y su posterior comprensión. Por esta razón, se les pidió a los docentes que se organizaran en mesa redonda para socializar y dialogar alrededor de diferentes cuestionamientos. Primero, se les preguntó si fueron claras las instrucciones descritas en el texto que elaboraron durante la actividad y si estas contribuyeron a la correcta construcción de la figura. Luego se indagó sobre las dificultades que se presentaron en el ejercicio y, finalmente, se preguntó acerca de dónde se podían encontrar este tipo de textos, qué características tienen los textos instructivos, para qué sirve la elaboración de estos y, por último, qué aprendieron sobre el texto instructivo.

Para el siguiente momento, "Construcción colectiva de conocimientos", se identificaron cada una de las etapas y las fortalezas de la clase modelo implementada. Las docentes investigadoras, junto al grupo de docentes reconstruyeron una a una las etapas de la clase modelo, con el fin de identificar las fortalezas de esta y develar cómo se configura una clase asentada en el modelo interaccionista. Para este propósito, se formularon las siguientes preguntas orientadoras: 1. ¿Qué les Ilamó la atención de la clase modelo? 2. ¿Qué fortalezas identificó en la clase modelo? 3. ¿Qué debilidades encontró en la clase modelo? 4. ¿Qué aspectos considera que se pueden mejorar?

La socialización-evaluación constituyó el último momento de ambos talleres, se logró la articulación de los conocimientos y habilidades adquiridas previamente para el análisis y reconstrucción de la clase modelo. En este momento se pretendía llegar a algunas conclusiones acerca de las concepciones que tiene el docente sobre la enseñanza de la escritura y la necesidad de buscar nuevas alternativas para el mejoramiento de este proceso en la escuela. El ejercicio reflexivo y la participación de los docentes cumplió un papel esencial en el desarrollo de esta última fase. Asimismo se realizó una entrevista, como técnica para recopilar información, para evaluar el cumplimiento de los objetivos de cada taller y las repercusiones en la forma de concebir la enseñanza por parte de los docentes. La evaluación se retroalimentó entonces con las percepciones de los docentes frente al alcance y desarrollo de la clase modelo, que favoreció que los docentes asumieran una actitud crítica, reflexiva y propositiva.

\section{Fase de reflexión}

Para llevar a cabo el análisis del corpus recolectado, por medio de la observación participante, el diario y la entrevista, se seleccionaron tres 
categorías que surgen de la aplicación de los talleres propuestos a 18 docentes de dos instituciones públicas de Bogotá. En la tabla 1, se presentan las categorías y subcategorías evidenciadas.

\section{Análisis y resultados}

\section{Concepciones y prácticas docentes para la enseñanza de la escritura}

Esta primera categoría surge de la triangulación de las observaciones, el registro de las intervenciones y las respuestas de los docentes, pues permitieron evidenciar las concepciones que ellos tienen sobre la enseñanza de la escritura.

Cuando se analizaron las respuestas dadas por los docentes a las preguntas formuladas durante el primer taller, se pudo evidenciar que la mayoría de los participantes concibieron la escritura como el aprendizaje del código alfabético, que se ve limitada al uso del alfabeto y a su asociación fonética. Así, por ejemplo, al preguntar acerca de la experiencia mediante la cual se tuvo acceso a la escritura, los docentes respondieron: "Aprendí primero conociendo las vocales, y luego la conformación con las letras, luego formando oraciones que tuvieran sentido para uno" (D1. Tomado de corpus talleres).

Tabla 1. Cuadro de categorías
Algunos docentes manifestaron que perciben la escritura como un proceso de transcripción (limitado a las grafías y su formación):

Me enseñaron escritura de manera silábica, con Nacho lee. Trazando puntos para formar la palabra. Rellenar palabras con diversos materiales. (D2. Tomado de corpus talleres)

Aprendí a escribir con métodos silábicos, a través del uso de cartillas como Nacho lee o Coquito, con el uso de cuaderno ferrocarril haciendo planas, decorando letras y repitiendo. (D3. Tomado de corpus talleres)

Varios recuerdan estas estrategias como algo agradable en su proceso de aprendizaje, por tanto consideran que este fue un método efectivo de enseñanza; sin embargo, otros docentes reconocen que esta forma de enseñar reduce la escritura a un uso prescriptivo, y descontextualizado.

Un mínimo porcentaje de docentes definió la escritura como una forma de representación del lenguaje: "Emplear la escritura como medio de comunicación de ideas de manera coherente" (D3. Tomado de corpus talleres). Esta variada forma de concebir la escritura representa las grandes diferencias que se dan frente a su enseñanza; así lo afirma Ferreiro (2002):

\begin{tabular}{|c|c|}
\hline Categoría general & Subcategoría \\
\hline \multirow{3}{*}{ Concepción sobre la enseñanza de la escritura } & Escritura como código alfabético \\
\hline & Escritura como copia o trascripción \\
\hline & Escritura como representación del lenguaje \\
\hline \multirow{4}{*}{ Prácticas docentes } & Un modelo tradicional \\
\hline & Un modelo condicionamiento operante \\
\hline & Un modelo constructivista \\
\hline & Un modelo sociocultural \\
\hline \multirow{4}{*}{ Enfoque sociocultural } & Papel del docente \\
\hline & Papel del estudiante \\
\hline & Objeto de enseñanza \\
\hline & Evaluación \\
\hline
\end{tabular}

Fuente: elaboración propia. 
La escritura puede ser conceptualizada de dos maneras muy diferentes $y$, según sea el modo en que se la considere, las consecuencias pedagógicas difieren drásticamente. La escritura puede ser considerada como una representación del lenguaje o como un código de transcripción de las unidades sonoras. (p. 13)

Es decir, al definir la escritura como habilidad de transcripción, su enseñanza se limita a las unidades sonoras (alfabeto) y a desarrollar asociaciones fonético-fonológicas, dejando de lado la función comunicativa, porque en los docentes prevalece una concepción de enseñanza de la lengua de forma inductiva, es decir de las partes al todo y manteniendo las estrategias pedagógicas con las cuales ellos aprendieron. Esto quizá se relacione con la falta de capacitación frente a otras perspectivas de enseñanza o la falta de actualización para tener conocimiento relacionado con nuevas estrategias pedagógicas que se pueden implementar en el aula con el propósito de desarrollar procesos significativos en la enseñanza de la lengua escrita. Como lo describe Vygotsky (1978):

[...] la escritura debería poseer un cierto significado para los niños, debería despertar en ellos una inquietud intrínseca y ser incorporada a una tarea importante y básica para la vida. Solo entonces podemos estar seguros de que se desarrollará no como una habilidad que se ejecuta con las manos y los dedos, sino como una forma de lenguaje realmente nueva y compleja. (p. 177)

\section{Prácticas docentes}

Desde la fundamentación teórica empleada en la elaboración de los talleres surge la categoría prácticas docentes. Durante la implementación del taIler 2, en la primera parte los docentes hicieron descripciones sobre sus prácticas pedagógicas. A partir de estas se estableció que algunas se correspondían con los modelos pedagógicos que se mostraron posteriormente.
Con relación a las prácticas en la enseñanza de la escritura, la mayoría de los docentes manifiestan que existen algunas ventajas al hacer uso de cartillas y la realización de planas, debido a que, según afirman, se logra la memorización de las letras y la construcción de sílabas. Este método, a su vez, permite mejorar la caligrafía, la ortografía y la gramática. Es así, como algunos docentes expresan: "El rol del estudiante es ser receptores y analistas..." (D4. Tomado de corpus talleres); "Trabajar con el uso de la cartilla permite desarrollar los conocimientos ortográficos y gramaticales del idioma español" (D5. Tomado de corpus talleres). Para Braslavsky (2003), esto se explica porque "la escritura era considerada como un hábito motor complejo, como un problema de renglones reglados o pautados. Se enseñaban letras pero no se enseñaba el lenguaje escrito" (p. 50)

De acuerdo con lo expuesto anteriormente, se puede evidenciar una concepción frente al uso y enseñanza de la lengua escrita desde el modelo tradicional, lo cual no permite reconocer el valor social y comunicativo que esta tiene, pues, como lo afirma Vygotsky (1978), "la enseñanza de la escritura se ha concebido en términos poco prácticos. Se ha enseñado a los pequeños a trazar letras y a formar palabras a partir de las mismas, pero no se les ha enseñado el lenguaje escrito" (p. 159). Por tanto, se demuestra que la forma en la cual se concibe la escritura repercute así mismo en las estrategias escogidas por los docentes para su enseñanza.

En este sentido, los docentes coinciden al afirmar que los estudiantes bajo este modelo pedagógico se convierten en receptores pasivos de lo que el docente transmite, y el maestro por su parte asume un rol autoritario en el aula, se considera el único dueño del conocimiento. La concepción de enseñanza desde este enfoque entiende el conocimiento como algo ya otorgado y cuya transmisión es magistral.

Otros docentes, por su parte, reconocen diversas desventajas en el aprendizaje de la escritura a través de la reiteración de estrategias mecánicas, 
que según mencionan, limitan la creatividad de los estudiantes debido a que tiende a presentarse el castigo ante el fallo y no se toma el error como una oportunidad para retroalimentar y mejorar diferentes aspectos del aprendizaje de la lengua escrita. Así mismo, no se evidencian procesos de comprensión ni se propicia el desarrollo del pensamiento, pues no se tiene una visión global de la palabra. También se reconoce que las cartillas son descontextualizadas, esto les impide a los estudiantes acceder al conocimiento de la lengua escrita de manera significativa.

A partir de los conceptos registrados en los talleres, se puede evidenciar que en la enseñanza de la escritura se ven reflejadas algunas prácticas desde el modelo constructivista, ya que los docentes encuentran que el papel de los estudiantes se hace más evidente al ser propositivos, autónomos e intercambiar conocimientos; mientras el docente es más un guía que orienta en la búsqueda de solución de problemas, invita a la reflexión y promueve la participación del estudiante. En este sentido la concepción pedagógica refleja un proceso de interacción entre el sujeto que aprende y los objetos de aprendizaje. El aprendizaje tiene como propósito la estructuración de nuevos esquemas cognitivos o transformación de los existentes, se tienen en cuenta los conocimientos previos con los que llega el estudiante a la escuela y se parte del contexto en el cual están inmersos. Flórez y Gómez (2012) los representa así:

El constructivismo psicogenético o cognitivista aportó una visión del proceso de aprendizaje del código escrito desde el punto de vista del niño que aprende a leer y a escribir, mostró la forma en que el niño va asimilando la información, las hipótesis que se plantea y las soluciones que genera el proceso. (p. 66)

Algunos docentes expresaron que el uso de premios como método de refuerzo logra estimular a los niños en el aprendizaje de la lengua escrita. Esto evidencia el modelo de condicionamiento operante, pues en algunas de las actividades que socializaron, pusieron de manifiesto la existencia de un refuerzo positivo o negativo dependiendo de que el niño escribiera de forma correcta o no. Un ejemplo de esto es el siguiente comentario: "Realicé un taller en el cual reforcé los temas trabajados durante la semana. cuando los niños respondían acertadamente les daba un dulce como premio, para motivarlos" (D5. Tomado de corpus talleres).

Hasta este punto se puede afirmar que, si bien es cierto que los docentes recurren a diferentes estrategias para enseñar la lengua escrita, no hay claridad frente a modelo pedagógico empleado; en algunos casos se integran diversos enfoques que conducen al eclecticismo y en el camino se tiende a perder el verdadero objetivo de enseñanza, pues no se tiene en cuenta el proceso requerido para favorecer el aprendizaje contextualizado y significativo de los estudiantes, quienes no se involucran durante la planificación y el desarrollo de las clases, lo cual conduce a pensar que aún se concibe al estudiante como un agente pasivo en su proceso de aprendizaje.

\section{Reflexiones sobre el quehacer docente, enfoque sociocultural}

En este apartado, se encuentran las experiencias y las reflexiones compartidas por los docentes en relación con lo que cada uno define frente a los modelos pedagógicos empleados en la enseñanza de la lengua escrita, de igual manera la percepción que tienen del papel del docente y objeto de enseñanza.

A partir de la clase modelo desde la perspectiva sociocultural propuesta en el desarrollo del segundo taller, se planteó a los docentes que identificaran el rol del docente, el del estudiante, el objeto de enseñanza y la evaluación tenida en cuenta en el proceso de enseñanza de la lengua escrita.

En el primer momento, la mayoría de los docentes presentan confusiones de carácter conceptual en relación con algunos términos relacionados con los procesos de enseñanza y aprendizaje; por 
ejemplo, existe una ambigüedad entre la definición de objeto de enseñanza y la metodología empleada en el desarrollo de las clases. Esto tal vez como resultado de que muchas de las prácticas docentes suelen estar influenciadas por las experiencias de enseñanza y aprendizaje de los maestros y no por su formación profesional y el nivel de capacitación y actualización que reciben es muy poco.

Algunos maestros asociaban el objeto de enseñanza con el proceso de aprendizaje definiéndolo como mecánico, propositivo. Otros, además, lo referencian como el contenido o el tema: "la carta", "uso de la $g$ y la $j$ ", entre otros. En esta medida, se desconocen los postulados de Vygotsky, quien manifiesta que el profesor no puede enseñar conceptos; sino que es tan solo un mediador entre los sujetos y objetos de aprendizaje. Se habla sobre la claridad frente al objeto de enseñanza, pues en primer momento no se tiene en cuenta a la hora de realizarlo, se queda en el hacer por hacer.

En la mayoría de las prácticas pedagógicas enfocadas en la enseñanza de la lengua escrita, socializadas por los docentes en el segundo taller, se permite identificar que estas carecen de un objeto de enseñanza claro, es decir, no hay un propósito de aprendizaje auténtico y contextualizado para los estudiantes; asimismo, no se ve reflejada en la descripción de las experiencias exitosas relatadas por los docentes una construcción teórica y conceptual que sirva de base en su quehacer como docentes. Esto contradice la posición que tiene la perspectiva sociocultural, así como lo plantea Fons (2011):

[...] en los planteamientos socioculturales además de tener en cuenta el texto y el sujeto, como elementos fundamentales del proceso de lectura y escritura, se toma en consideración el contexto, es decir la forma como el entorno social y cultural incide en la comprensión de la lectura, en la producción de textos y en los usos que las personas hacen del lenguaje escrito. (p. 4)

De igual manera, llamó la atención que ningún docente de las dos instituciones hiciera referencia a cómo se llevó a cabo la evaluación de los procesos de enseñanza y aprendizaje de la escritura. Esto permite pensar que aun el docente dentro de su planeación de clase no tiene en cuenta la evaluación como elemento primordial y que probablemente lo deja como una fase final en el proceso. De acuerdo con ello, al no tener un propósito definido, la evaluación queda relegada a la verificación, perdiendo así su propósito pues "la evaluación constituye una oportunidad excelente para que quienes aprenden pongan en práctica sus conocimientos y se sientan en la necesidad de defender sus ideas, sus razones, sus saberes" (Álvarez, 2005, p. 13), es decir, es el poder poner en práctica aquello que se aprendió.

Otro resultado que arroja la socialización de un modelo de clase es que el docente no parte de problemas reales, ni tiene en cuenta los intereses de los estudiantes. Esto se evidenció gracias a que no se realizó una descripción detallada de la población, ni se presentó una contextualización previa. En consecuencia, no se logró establecer cuáles son los criterios del docente para desarrollar unas competencias específicas de la lengua y no otras de acuerdo con las características particulares de los grupos de estudiantes.

A pesar de lo anterior, la mayoría de los docentes presentaron aportes conceptuales que sustentan su práctica, como también resaltan el rol del docente como mediador y guía durante las actividades de enseñanza y aprendizaje en el aula, incluso mencionan la importancia de las estrategias de metacognición en el aprendizaje de la escritura y se reconoce que esta se debe trabajar como un proceso y no como un producto. Además, cabe destacar que en el desarrollo de las actividades que comparten los docentes se le da prelación al trabajo colaborativo.

\section{Conclusiones}

Los docentes que atienden a la población del ciclo inicial manifiestan que debe haber una actualización permanente y una formación encaminada a 
encontrar y desarrollar estrategias nuevas y efectivas en la enseñanza de la escritura, atendiendo a los cambios en las políticas públicas educativas, como también a los desafíos actuales desde lo político y social. Por tanto, es necesario brindar espacios de formación, discusión y reflexión que posibiliten un mejoramiento en el quehacer docente.

En el desarrollo de los talleres se evidenció que los docentes acuden a los mismos métodos con los que aprendieron a escribir desde un enfoque prescriptivo, cuya orientación está basada en la repetición y la memoria, incluso encuentran que estrategias como las planas y el uso de cartillas fueron fundamentales en la adquisición del código. Sin embargo, hacen alusión a la importancia de generar estrategias que permitan desarrollar procesos significativos en la enseñanza de la lengua escrita. Entonces, se podría concluir que falta coherencia entre el discurso del docente frente a la metodología que aceptan como propicia para la enseñanza de la escritura y las prácticas pedagógicas que se llevan a cabo en el aula.

A pesar de que en el decir los docentes manifiestan la importancia de reconocer nuevas teorías y enfoques en cuanto a la enseñanza y aprendizaje de la escritura, en el hacer se evidencia que esta sigue siendo vista como una habilidad motriz que se reduce al uso prescriptivo. Esto se refleja en las prácticas que se viven día a día en la escuela, seguramente condicionadas por las concepciones docentes que subyacen a este proceso, debido a que están construidas a partir de la propia experiencia de los docentes, el conocimiento teórico que poseen, las creencias y, en general, las ideas que acompañan sus prácticas pedagógicas.

Uno de los aspectos a resaltar de las prácticas docentes es que, en las actividades de clase, "se les permite a los estudiantes tener un papel activo y participativo"; sin embargo, para el desarrollo de estas, se evidencia un total desconocimiento de la población, sus intereses y, en consecuencia, de sus necesidades. Así, pues, los propósitos no son coherentes con las características específicas de la población, como tampoco las actividades propuestas.
Finalmente, con la implementación de los talleres se corrobora la necesidad de una formación continua que apunte a la movilización de las concepciones a través del diálogo, la reflexión y el análisis de las prácticas en el aula. Esto con el fin de mejorar las estrategias pedagógicas, ya que, como se recoge en las apreciaciones dadas por los docentes frente a los talleres implementados, es importante generar espacios de actualización e intercambio de saberes que posibiliten una continua reflexión relacionada con los procesos de enseñanza y aprendizaje de la escritura que se llevan a cabo en las diferentes instituciones.

\section{Reconocimientos}

Este artículo surge como respuesta a los objetivos planteados en el interior del grupo de investigación Oralitura, en el que se buscó contribuir al mejoramiento de las prácticas pedagógicas en la modalidad de escritura, a través de la planeación, diseño e implementación de diversos talleres dirigidos a docentes.

Este grupo de investigación fue creado en el marco del Seminario de Líneas de Investigación, de la Maestría en Pedagogía de la Lengua Materna, de la Universidad Distrital Francisco José de Caldas.

\section{Referencias bibliográficas}

Álvarez, J. (2005). Evaluar para conocer, examinar para excluir. Madrid: Ed. Morata.

Braslavsky, B. (1997). La escuela puede. Una perspectiva didáctica. Buenos Aires: Aique.

Braslavsky, B. (2003). ¿Primeras letras o primeras lecturas? Una introducción a la Alfabetización temprana. Buenos Aires: Fondo de Cultura Económica.

Cassany, D. (1999). Construir la escritura. Barcelona: Paidós.

Contreras. D. (1994). La investigación en la acción. Cuadernos de Pedagogía, 224, 8-12.

Díaz Barriga, Frida. (2005). Enseñanza situada: Vínculo entre la escuela y la vida. México: McGraw Hill. 
Díez, C. (2004). La escritura colaborativa en educación infantil. Estrategias para el trabajo en el aula. Barcelona: Ed. Horsori.

Elliot, J. (2000). La investigación acción en educación. Madrid: Ed. Morata.

Ferreiro, E. (2002). Alfabetización. Teoría y práctica. Buenos Aires: Siglo XXI Editores.

Flick, U. (1998). Introducción a la investigación cualitativa. Madrid: Ed. Morata.

Flórez, R. y Gómez, D. (2013). Leer y escribir en los primeros grados: retos y desafíos. Bogotá: Universidad Nacional de Colombia.

Fons, M. (2011). Enseñar a leer para vivir. Barcelona: Universidad de Barcelona. Recuperado de b10. berritzeguneak.net/eu/descargar_fichero.php?file=leer_para_vivir.pdf

Goodman, K. (1990). El lenguaje integral: un camino fácil para el desarrollo del lenguaje. Recuperado de http://www.lecturayvida.fahce.unlp.edu.ar/numeros/a11n2/11_02_Goodman.pdf

Jaimes, G. (2008). Modelos epistemológicos, pedagógicos y lingüísticos en la enseñanza de la lengua materna. En Aprender a dialogar en el aula de preescolar (pp. 19-25). Bogotá: Universidad Distrital Francisco José de Caldas.

Kemmis, S. (1988). El currículum más allá de la teoría de la reproducción. Madrid: Morata.

Lerner, D. (2001). Leer y escribir en la escuela: lo real, lo posible y lo necesario. México: Fondo de Cultura Económica.
Ministerio de Educación Nacional (MEN) (1998). Lineamientos Curriculares de Lengua Castellana. Bogotá: Cooperativa Editorial Magisterio.

Morales, R. y Bojacá, B. (2000). Relación entre concepciones sobre el lenguaje y la lengua escrita. Enunciación, 4(1), 109-118. DOI: https://doi. org/10.14483/22486798.2541

Pérez, A., Julián, J. A. y López, V. (2009). Evaluación formativa y compartida en el Espacio Europeo de Educación Superior. En V. López (coord.), Evaluación formativa y compartida en Educación Superior. Propuestas, técnicas, instrumentos y experiencias (pp. 19-44). Madrid: Narcea Ediciones. Recuperado de https://books.google.com.co/books?id=dz2CZ5zPInUC\&printsec $=$ frontcover\&hl=es $\# \mathrm{~V}=$ onepage\&q\&f=false

Rodríguez, M. (2012). El taller: una estrategia para aprender, enseñar e investigar. En S. Soler (comp.), Lenguaje y educación: perspectivas metodológicas y teóricas para su estudio (pp. 13-43). Bogotá: Universidad Distrital Francisco José de Caldas, Universidad del Valle, Universidad Pedagógica Nacional.

Roselli, N. (2016). El aprendizaje colaborativo: bases teóricas y estrategias aplicables en la enseñanza universitaria. Propósitos y Representaciones, 4(1), 219-280. DOI: http://dx.doi.org/10.20511/ pyr2016.v4n1.90

Vygotsky, L. (1978). El desarrollo de los procesos psicológicos superiores. Barcelona: Crítica. 